\title{
Role of volume versus defects in the electrical resistivity of lattice-distorted V(001) ultrathin films
}

\author{
Y. Huttel, ${ }^{1}$ J. I. Cerdá, ${ }^{1}$ J. L. Martínez, ${ }^{1}$ and A. Cebollada ${ }^{2}$ \\ ${ }^{1}$ Instituto de Ciencia de Materiales de Madrid, Consejo Superior de Investigaciones Científicas (CSIC), \\ C/ Sor Juana Inés de la Cruz, 328049 Madrid, Spain \\ ${ }^{2}$ Instituto de Microelectrónica de Madrid-IMM (CNM-CSIC), Isaac Newton 8 (PTM), 28760 Tres Cantos, Madrid, Spain
}

(Received 2 August 2007; published 30 November 2007)

\begin{abstract}
$4 \mathrm{~nm}$ thick V layers grown by triode sputtering on $\mathrm{MgO}(001)$ single crystals and capped with $\mathrm{MgO}$ exhibit a perfect epitaxy accompanied by a tetragonal distortion and an unexpected volume compression that increases with the $\mathrm{V}$ deposition temperature. The electrical resistivity follows a deposition temperature dependence with these structural modifications, decreasing by an order of magnitude across the temperature range studied. Total energy $a b$ initio calculations rule out electronic structure changes and/or oxygen interface diffusion as responsible for the structure variation. Calculations of the ballistic conductance for the epitaxial $\mathrm{V}$ films do not reproduce the resistivity-volume correlation, implying a diffusive electron transport mechanism in the films, despite their high crystallinity. Instead, we assign the origin of the electrical behavior to the presence of growth induced defects in the $\mathrm{V}$ lattice, whose density is higher in films deposited at low temperature, and decreases as deposition temperature increases. These results extend the previous findings in volume expanded $\mathrm{H}$ loaded $\mathrm{Fe} / \mathrm{V}$ and $\mathrm{Mo} / \mathrm{V}$ superlattices to simpler structures where the $\mathrm{H}$ content is negligible and, additionaly, all the electronic transport is confined within the $\mathrm{V}$ film.
\end{abstract}

DOI: 10.1103/PhysRevB.76.195451

PACS number(s): 68.55.Jk, 73.61.-r, 31.15.Ar, 71.15.Mb

\section{INTRODUCTION}

The study of the properties of ultrathin and nanostructured films is motivated by both fundamental reasons and potential technological applications. Subtle modifications of the physical properties of these systems are achieved by tiny changes of the structure that, for example, can be induced by adsorption or insertion of doping elements such as H. Vanadium, which is known to act as a strong hydrogen getter, ${ }^{1-4}$ is the natural prototype element to study the effects of introducing $\mathrm{H}$ in very thin structures and nanoparticles. Furthermore, these systems are attracting attention for the design of new materials for information storage and hydrogen storage for energetic applications. ${ }^{5,6}$ Very recently, Meded et al. ${ }^{7}$ have reported in-plane resistivity variations upon hydrogen loading of $\mathrm{Fe} / \mathrm{V}$ and $\mathrm{Mo} / \mathrm{V}$ superlattices. They have interpreted their findings in terms of electronic structure modifications via hydrogen induced $\mathrm{V}$ expansion, with no contribution to the resistivity of the hydrogen atoms. They have concluded that the sole physical effect behind the modification of the resistivity of the multilayers is the vanadium volume expansion resulting from the incorporation of $\mathrm{H}$.

In view of the interest in these results, we have followed a different approach to study this phenomenon, producing volume distorted $\mathrm{V}$ ultrathin films without the inclusion of either an external element responsible of the lattice expansion $(\mathrm{H})$ or additional conducting layers $(\mathrm{Fe}$ and $\mathrm{Mo})$ that might contribute to the resistivity of the whole system. In addition, we study these effects not only in volume expanded systems, but also in both expanded and compressed ones. We present results on the correlation of the electrical resistivity with the volume expansion and compression of $\mathrm{V}$ ultrathin films grown at different temperatures in $\mathrm{MgO} / \mathrm{V} / \mathrm{MgO}(001)$ fully epitaxial trilayers by triode sputtering. Here, the only layers that contribute to the resistivity measurements are the $\mathrm{V}$ layers, making, in principle, the interpretation of the resis- tivity results more straightforward. Also, the lattice modification of the $\mathrm{V}$ layers is not induced by $\mathrm{H}$ loading, but rather controlled by the growth conditions of the structures as reported below.

Additionaly, we have performed density functional theory $^{8}$ (DFT) based calculations exploring the impact that interface vanadium oxide layers exert on the volume of the $\mathrm{V}$ films. We have also calculated the ballistic conductance of these films in order to check if a defect-free atomistic description of the system could account for the measured volume-resistivity correlation.

\section{EXPERIMENTAL DETAILS}

All the samples were grown in an ultrahigh vacuum system with triode sputtering and laser ablation facilities. The ultrathin V films (4 nm thick) were deposited by triode sputtering at different temperatures on $\mathrm{MgO}(001)$ single crystals. The argon pressure during sputtering was $4 \times 10^{-4} \mathrm{mbar}$ and the base pressure was in the low $10^{-9}$ mbar. The sputtering voltage was $1 \mathrm{kV}$ and the resulting deposition rate was $0.4 \AA /$ min. Prior to V deposition, a $10 \mathrm{~nm} \mathrm{MgO}$ buffer layer was grown by laser ablation at $450{ }^{\circ} \mathrm{C}$ on the substrate to planarize the surface. ${ }^{9}$ Subsequently, a $10 \mathrm{~nm}$ thick $\mathrm{MgO}$ capping layer was deposited at room temperature (RT) to protect the $\mathrm{V}$ layers from oxidation. A great advantage of these kind of structures is that the only metallic layer is the $\mathrm{V}$ film under investigation, the substrate, buffer, and capping layers being insulators. This is very convenient to directly measure the electrical resistivity of the V layer ex situ without shorting effects from metallic capping or adjacent layers. The crystallographic structure of the samples was studied $e x$ situ by x-ray diffraction (XRD) using a four-circle diffractometer, $K \alpha$ radiation, and $1 / 4^{\circ}$ divergence slits. The electrical resistivity was measured using a four-probe setup in the temperature range from 10 to $300 \mathrm{~K}$. Electrical contacts were 


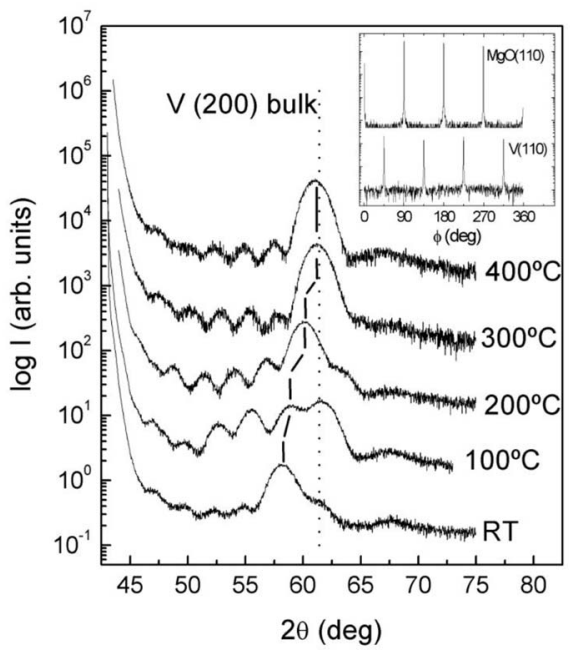

FIG. 1. XRD symmetric scans for the $\mathrm{MgO} / \mathrm{V} / \mathrm{MgO}(001)$ structures with $\mathrm{V}$ deposition temperature ranging from $\mathrm{RT}$ up to $400{ }^{\circ} \mathrm{C}$. Inset: Representative asymmetric $\phi$ scans at V (110) and $\mathrm{MgO}$ (110) peaks showing the epitaxial nature of the $\mathrm{V}$ layers and the $45^{\circ}$ in-plane rotation of the $\mathrm{V}$ lattice with respect to the $\mathrm{MgO}$ one.

applied on the $\mathrm{V}$ layer by scratching the insulating capping layer until electrical continuity was achieved.

\section{EXPERIMENTAL RESULTS}

The detailed structural characterization of the samples has been presented elsewhere, ${ }^{10}$ and here, we only describe the main aspects that are relevant in this work. In Fig. 1, we show the high angle symmetric XRD scans for $\mathrm{MgO} / \mathrm{V} / \mathrm{MgO}(001)$ structures, where the $\mathrm{V}$ layers have been grown at temperatures ranging from RT up to $400{ }^{\circ} \mathrm{C}$. The high intensity peak tail below $45^{\circ}$ corresponds to the $\mathrm{MgO}(200)$ reflection. In all spectra, the $\mathrm{V}(200)$ peak is observed together with clear Kiessig fringes, characteristic of a high quality growth, with sharp interfaces and structural coherence length equivalent to the total V layer thickness. This last point was confirmed by applying Scherrer's equation to the (200) diffraction peak, obtaining a structural coherence length along the growth direction of $4 \mathrm{~nm}$, equivalent to the total thickness of the V layer, for all growth temperatures. ${ }^{10}$

$\mathrm{X}$-ray reflectometry measurements (not shown here) confirm the sharp character of the interfaces with well defined oscillations, and atomic force microscope measurements on $\mathrm{MgO}$ capped and uncapped $\mathrm{V}$ samples deposited at the same temperatures yielded rms roughness of around $3 \AA .{ }^{10}$ It can be observed in the spectra of Fig. 1 that the V(200) peak appears clearly displaced from its bulk position, with a gradual shift toward smaller scattering angles with decreasing deposition temperature. Asymmetric (111) and (110) V reflections where also detected, confirming the $\mathrm{V}(001)$ $\times[100] \| \mathrm{MgO}(001)[110]$ epitaxial relationship. This is illustrated in the inset of Fig. 1, where azimuthal $\phi$ scans for both (110) $\mathrm{V}$ and $\mathrm{MgO}$ reflections are presented, showing the $45^{\circ}$ in-plane rotation of the $\mathrm{V}$ lattice with respect to the $\mathrm{MgO}$,
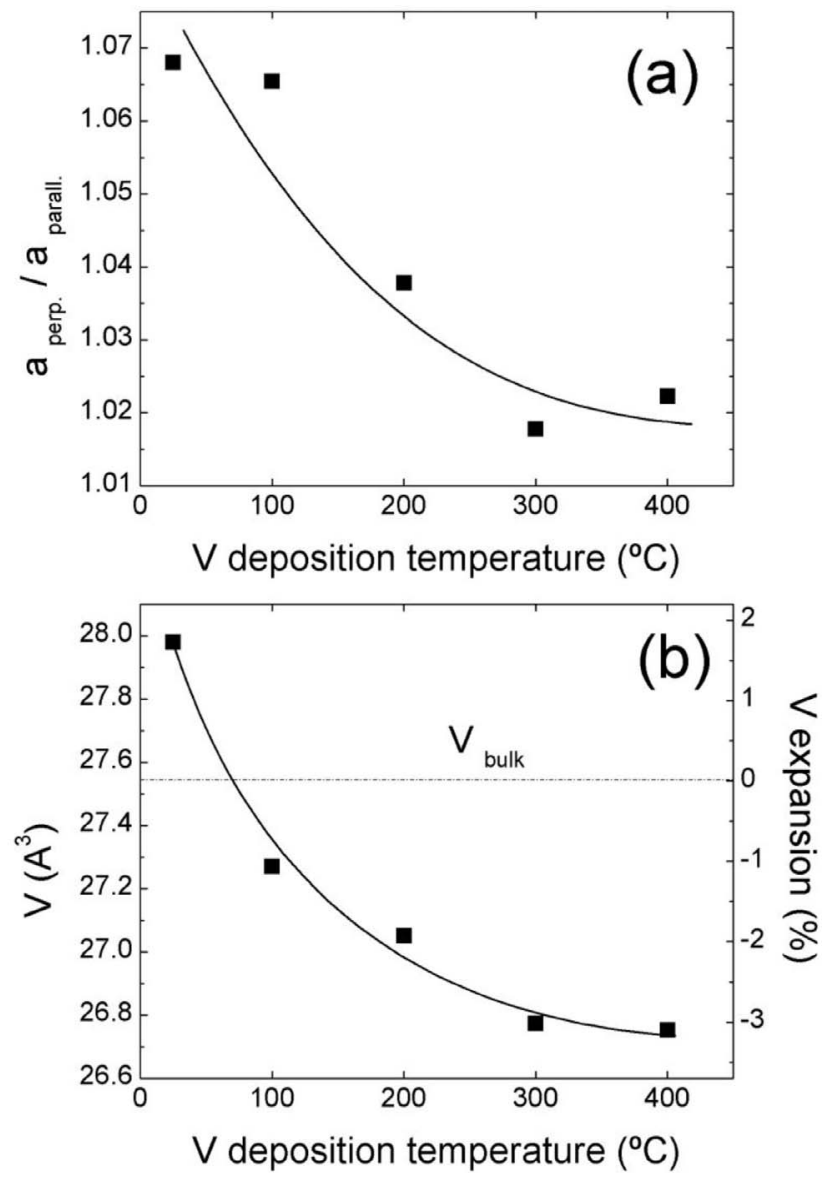

FIG. 2. (a) Tetragonal distortion $\left(a_{\perp}^{V} / a_{\|}\right)$versus $\mathrm{V}$ deposition temperature. (b) V volume cell (left axis) and relative change (right axis) as a function of $\mathrm{V}$ deposition temperature (lines are guides for the eye).

with the $\mathrm{V}$ atoms sitting on top of $\mathrm{O}$ atoms. ${ }^{11}$ Both in-plane and out-of-plane $\mathrm{V}$ lattice parameters, $a_{\perp}^{V}$, were determined through the positions of the (200), (211), and (110) V reflections for the different structures considered in this work. We find that the $\mathrm{V}$ grows under in-plane compression, having a coherent interface with the $\mathrm{MgO}$, as the $45^{\circ}$ in-plane lattice rotation implies an almost perfect match with the $\mathrm{MgO}$ lattice. In fact, the in-plane $\mathrm{V}$ lattice parameters $(\approx 2.96 \AA)$ are almost equal to the $\sqrt{2}$ corrected $\mathrm{MgO}$ parameter $(2.97 \AA)$. On the other hand, the out-of-plane V lattice parameter for films deposited at RT, $100{ }^{\circ} \mathrm{C}$, and $200^{\circ} \mathrm{C}$ appears expanded with a higher expansion for RT deposition, adopting the bulk value for samples grown at 300 and $400{ }^{\circ} \mathrm{C}$.

From the measured in-plane and out-of-plane lattice parameters, we have determined the tetragonal distortion $\left(a_{\perp}^{V} / a_{\|}\right)$and volume of the $\mathrm{V}$ lattice as a function of deposition temperature that are presented in Fig. 2. The tetragonal distortion is found to be larger than one over the whole $\mathrm{V}$ deposition temperature range, with a maximum value of almost $7 \%$ for RT deposition, decreasing as deposition temperature increases, and reaching values around $2 \%$ for 300 and $400{ }^{\circ} \mathrm{C} \mathrm{V}$ deposition temperatures. Regarding the volume expansion, it also shows a continuous variation with 

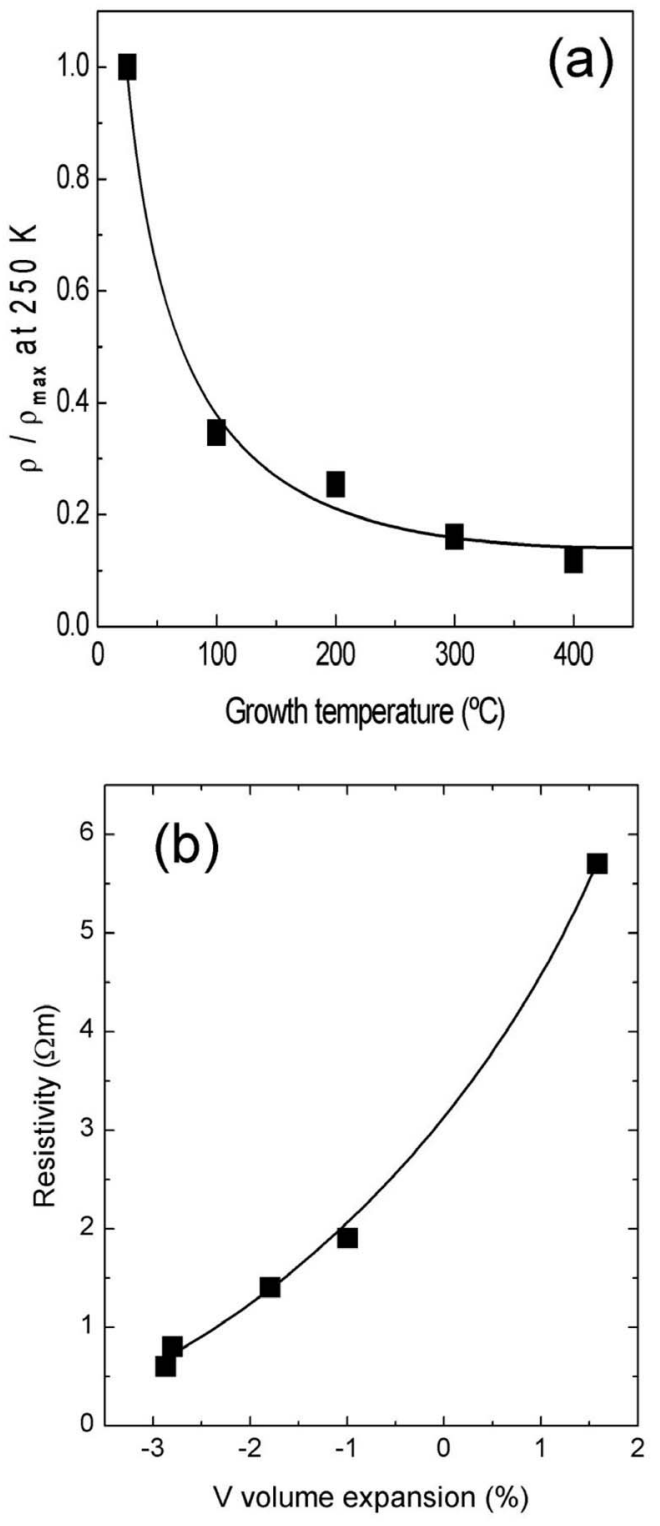

FIG. 3. (a) $\mathrm{V}$ resistivity as a function of the $\mathrm{V}$ deposition temperature. (b) $\mathrm{V}$ resistivity as a function of $\mathrm{V}$ volume modification (lines are guides for the eye).

deposition temperature, with a maximum value (around 2\%) for RT deposition and decreases as deposition temperature increases. Remarkably, we find an inversion in the sign of the volume expansion as the deposition temperature increases, being positive for RT deposition, but negative for all the other deposition temperatures, turning into a gradually increasing compression as deposition temperature increases, with a maximum value of $-3 \%$ for $400{ }^{\circ} \mathrm{C}$.

We now turn to the electrical characterization of the same series of $\mathrm{V}$ thin layers as a function of deposition temperature and, therefore, as a function of the volume distortion. The results are summarized in Fig. 3. In Fig. 3(a), we show the evolution of the resistivity of the $\mathrm{V}$ layers measured at $250 \mathrm{~K}$ as a function of the growth temperature. The resistivity values have been normalized to the maximum resistivity measured for the V thin film grown at RT. As can be ob- served, there is a continuous decrease of the resistivity as the deposition temperature increases. Since we have previously shown the direct correlation between deposition temperature and volume expansion, in Fig. 3(b) we show the volume expansion dependence of the $\mathrm{V}$ resistivity in this system, where a clear and continuous dependence of the resistivity as a function of the volume expansion can be observed, regardless of the sign of the latter, i.e., regardless if the $\mathrm{V}$ lattice is compressed or expanded. These results are similar to those obtained in $\mathrm{H}$ loaded structures, although we here extend the $\mathrm{V}$ volume variation to negative values, thus covering both compression and expansion situations.

Interestingly, we find that not only the trends observed in the evolution of the resistivity of the $\mathrm{V}$ layers are similar to those reported for $\mathrm{V} / \mathrm{Fe}$ and $\mathrm{V} / \mathrm{Mo}$ multilayers, i.e., the resistivity increases with increasing $\mathrm{V}$ lattice volume, but also that the changes in the resistivity are of the same order. In fact, here we find that a modification of the $\mathrm{V}$ lattice volume of $5 \%$ corresponds to a change of resistivity by approximately a factor of 10 . Recently, Burkert et al. ${ }^{12}$ found that the average expansion of the out-of-plane lattice parameter of $\mathrm{V}$ in $\mathrm{Fe} / \mathrm{V}$ multilayers loaded with $50 \% \mathrm{H}$ is close to $5 \%$. They also found that the in-plane lattice parameter was not modified by $\mathrm{H}$ loading, leading to a volume expansion close to $5 \%$. Assuming that $0.8<\Delta \rho / \Delta \rho_{\max }<0.9$ for $50 \% \mathrm{H}$ loaded $\mathrm{Fe} / \mathrm{V}$ multilayer, ${ }^{7}$ this corresponds to a resistivity change for this system that is close to a factor of 6 , i.e., not far from the results presented here.

Since the resistivity changes are similar to those observed in $\mathrm{H}$ loaded structures, it is important to discuss the possible origin of the $\mathrm{V}$ lattice distortion, in our case, its impact on the resistivity of the thin $\mathrm{V}$ layers and also the presence of hydrogen in the $\mathrm{V}$ layers. Even though there was no intentional addition of hydrogen in the $\mathrm{V}$ lattice in the present work, the lattice distortion could be due to residual hydrogen, because of the strong affinity of $\mathrm{V}$ to absorb $\mathrm{H}$, and the presence of $\mathrm{H}$ in the growth chamber since this element is always present in measurable amounts even in ultrahigh vacuum systems. In order to clarify this important point, we have performed elastic recoil detection analysis measurements ${ }^{13}$ for samples exhibiting extreme changes in lattice parameter (expansion and compression). For all samples, a residual $\mathrm{H}$ content $\leq 1 \%$ was detected, which is clearly below the $\mathrm{H}$ concentration needed to modify the lattice parameters of $\mathrm{V}^{7}$ These results rule out the $\mathrm{H}$ contamination as responsible for the $\mathrm{V}$ lattice distortion, and favor other mechanisms.

\section{THEORETICAL RESULTS}

The results presented in the previous section show a striking behavior of the volume $V$-or equivalently $a_{\perp}^{V}$-with the annealing temperature. Here, we address the possibility that the $\mathrm{V}$ lattice distortion stems from subtle modifications of the $\mathrm{V} / \mathrm{MgO}(001)$ interface, which can be produced by different growth temperatures. ${ }^{13}$ It is reasonable to expect that issues such as the composition of the first $\mathrm{V}$ layers in contact with the $\mathrm{MgO}(001)$ substrate and their relative registry might result in different $\mathrm{V}$ thin film structures. Therefore, we have 


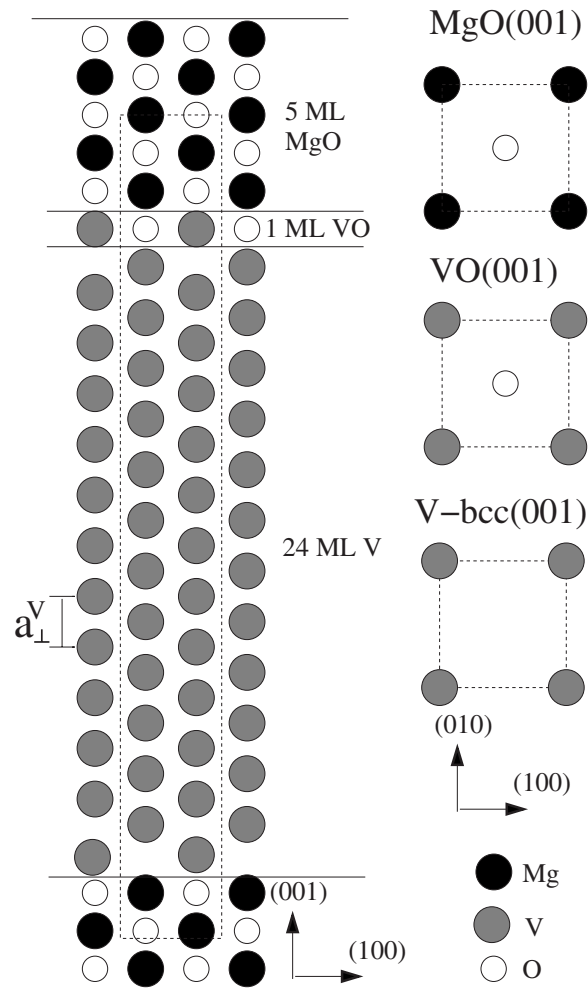

FIG. 4. Top and side views of the models employed in the simulations for the embedded $\mathrm{V}$ films. The left figure corresponds to an asymmetric $(\mathrm{MgO})_{5} /(\mathrm{VO})_{1} /(\mathrm{V})_{24} /(\mathrm{VO})_{0} /(\mathrm{MgO})_{5}$ structure. The dashed rectangle shows the unit cell. The interlayer $\mathrm{V}$ spacing $a_{\perp}^{V}$ is also indicated although we actually compute it as the average interlayer spacing between the oxygen-free $\mathrm{V}$ layers. Top views on the right give the in-plane structure for the different types of layers considered in this work

theoretically explored, at the atomic level, the existence of (meta)stable phases possibly induced by the creation of thin vanadium oxide layers at the $\mathrm{MgO} / \mathrm{V}$ interface, which could present different $\mathrm{V}$ atomic volumes within the metallic film.

\section{A. Structural analysis}

The coherent nature of the $\mathrm{V} / \mathrm{MgO}$ interface as well as the crystalline coherency along the growth direction makes the $\mathrm{V}$ film rigidly bonded to the substrate in the in-plane direction, with only the vertical direction free to modify the lattice parameter. Besides, since the capping layer is also $\mathrm{MgO}$, we can assume the upper $\mathrm{MgO} / \mathrm{V}$ interface to be also coherent, implying a strong structural constriction for the $\mathrm{V}$ film. We have modeled such scenario embedding a 25 atom thick V film between five $\mathrm{MgO}$ layers in a multilayer geometry, as depicted in Fig. 4.

The metallic and oxide films follow bcc and rocksalt stackings, respectively, and they are both (001) oriented. A perfect epitaxy is assumed between the two films, so that the system may be laterally described with a squared $p(1 \times 1)$ unit cell (see top views on the right of Fig. 4). Apart from the clean interface case, we have also considered oxygen-rich interfaces by adding one or two oxygen atoms to the $\mathrm{V}$ planes closer to the $\mathrm{MgO}$, i.e., substituting the $\mathrm{V}$ atomic planes by VO planes. Thus, our model systems may be denoted by $(\mathrm{MgO})_{5} /(\mathrm{VO})_{m} /(\mathrm{V})_{n} /(\mathrm{VO})_{m^{\prime}} /(\mathrm{MgO})_{5}$, with $n+m$ $+m^{\prime}=25$ fixed. The $m=m^{\prime}=0$ case represents the clean interface, $m=m^{\prime}$ leads to symmetric interfaces, and $m^{\prime}=0$ corresponds to a clean interface at the top, i.e., no oxygen diffusion at the top $\mathrm{MgO}$ capping layer. Additionally, there are two possible high-symmetry stackings at each interface: a metal atom is placed below or on top of an oxygen atom or below or on top of another metal atom. In the case of symmetric systems, this leads to two possible stacking configurations for $m=0$, three for $m=1$, and up to eight for $m=2$.

We have employed the pseudopotential ${ }^{14}$ DFT based SIESTA code ${ }^{15}$ for all total energy calculations. A linear combination of atomic orbitals is used as basis set, while an $(8$ $\times 8 \times 1) k$ sampling was applied to the three-dimensional cell. In choosing the exchange-correlation functional, we checked the performance of both the local density approximation $^{16}$ (LDA) and the generalized gradient approximation ${ }^{17}$ (GGA). LDA yielded rather contracted lattice parameters for both the ceramic and V-bcc bulk phases $\left[a^{L D A}(\mathrm{MgO})=4.13 \AA\right.$ vs $a^{\exp }(\mathrm{MgO})=4.21 \AA$ and $a^{L D A}(\mathrm{~V})$ $=2.89 \AA$ vs $\left.a^{\exp }(\mathrm{V})=3.02 \AA\right]$. The GGA scheme, on the contrary, provided good agreement for the metallic phase, but an expanded value for the oxide $\left[a^{G G A}(\mathrm{~V})=3.06 \AA\right.$ and $\left.a^{G G A}(\mathrm{MgO})=4.35 \AA\right]$. Geometry optimizations via total energy minimization were carried out for our model systems, allowing all atoms to relax as well as the total thickness of the unit cell in order to remove any stress along the system normal. The in-plane lattice parameter $a_{\|}$was fixed to $2.97 \AA$ in all cases. As a reference, we considered a tetragonally distorted V-bcc bulk phase, with the in-plane lattice parameter also fixed to $2.97 \AA$, while $a_{\perp}^{V}$ was swept over a wide range of values; we will refer to this latter system as the V-bct bulk phase. We note that the self-consistent procedure implemented in SIESTA ${ }^{15}$ is particularly problematic for inhomogeneous systems containing $3 d$ transition metals, resulting in very long computation times. We were, thus, forced to reduce the accuracy of the SIESTA calculation parameters, e.g., single zeta $d$ atomic orbitals for $\mathrm{V}$, or to neglect any interactions between nonoverlapping atoms. We have, nevertheless, checked for some test cases that our qualitative conclusions do not vary if more accurate calculations are performed instead. At the same time, a systematic study involving all possible structures became computationally intractable. Still, we have covered a wide spectrum of configurations: 13 and 12 different cases for the LDA and GGA calculations, respectively.

As outcome of this theoretical study, we found that, for all structures, the interlayer spacing $a_{\perp}^{V}$ never showed strong deviations with respect to the value optimized for the bulk V-bct. We plot in Fig. 5 the GGA derived total energy differences and pressures as a function of $a_{\perp}^{V}$ for the V-bct case as well as for the most stable sandwiched $\mathrm{V}$ films found. The total energy minima are attained close to $a_{\perp}^{V}=3.20 \AA$, which is $0.05 \AA$ smaller than the expected value of $a_{\perp}^{V}=3.25 \AA$ if the GGA atomic volume of vanadium is preserved (dashed vertical line in the plots). The rest of the structures showed deviations from this value always smaller than $\pm 0.02 \AA$. 


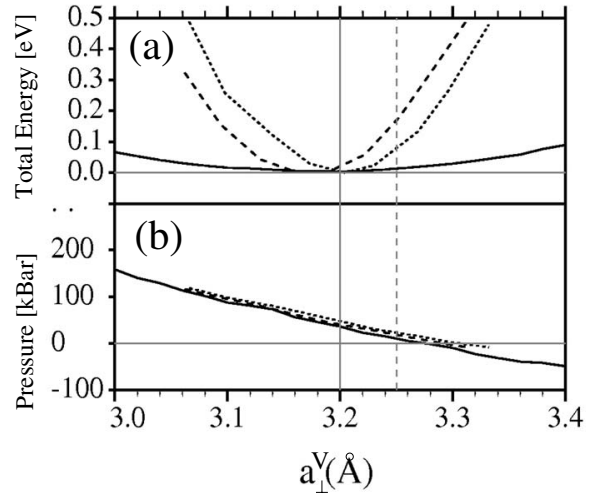

FIG. 5. (a) Total energy differences as a function of the V interlayer spacing $a_{\perp}^{V}$ for the V-bct bulk phase (solid lines), the $(\mathrm{MgO})_{5} /(\mathrm{VO})_{0} /(\mathrm{V})_{25} /(\mathrm{VO})_{0} /(\mathrm{MgO})_{5}$ system (dashed line), and the $(\mathrm{MgO})_{5} /(\mathrm{VO})_{1} /(\mathrm{V})_{23} /(\mathrm{VO})_{1} /(\mathrm{MgO})_{5}$ one (dotted line). (b) Total pressure as a function of $a_{\perp}^{V}$ for the same cases as in (a). The solid vertical line corresponds to the average equilibrium value $\left\langle a_{\perp}^{V}\right\rangle$ obtained for all the systems studied under the GGA. The dashed vertical line gives the value of $a_{\perp}^{V}$ at which the GGA atomic volume of $\mathrm{V}$ is retrieved.

Hence, the $\mathrm{V}$ films always adopt a $a_{\perp}^{V}$ value which leads to a $1.5 \%$ compression of the theoretically optimized volume for the ideal V-bcc phase, regardless of the interface configuration or the presence of interface VO layers. We believe that this is a general result since it also applies to unstable structures with total energies up to $4 \mathrm{eV}$ above the most stable ones. The LDA results, not shown here, were similar to the GGA case, except that a $2 \%$ expansion is found instead of a volume compression. However, we feel that the GGA results are more reliable since they reproduce more closely the V-bcc structural properties.

In the lower part of the figure, we plot the evolution of the pressure as a function of $a_{\perp}^{V}$. At equilibrium spacing, there is no stress along the normal (001) direction, but a residual pressure exists, which stems from the in-plane stress imposed on the films. As the interlayer spacing is increased, this in-plane stress is released although it involves an energy cost. Therefore, our main conclusion of the present structural analysis is that the most stable structure of the in-plane strained $\mathrm{V}$ films is not the one which preserves the volume in the bcc phase, but the films that suffer a small contraction $(\sim 1.5 \%)$ along the normal direction. Further, interface effects such as oxygen diffusion at the interface or the existence of metastable configurations has no impact on the volume compression.

\section{B. Ballistic conductance}

In this section, we try to rationalize the resistivitycompression relationship evidenced in Fig. 3. We have retained the quantum atomistic picture together with the model systems employed in the previous section, and calculated the conductance of such films along the in-plane (100) direction. Imposing a perfect epitaxy implies that the geometries are defect-free and periodic along the electron transport direc-

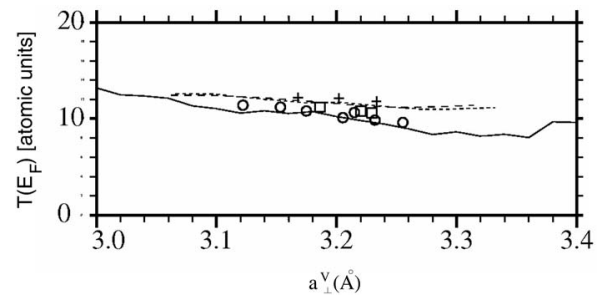

FIG. 6. The transmission coefficient $T\left(E_{F}\right)$ along the (100) direction as a function of the $\mathrm{V}$ interlayer spacing $a_{\perp}^{V}$ for the bulk V-bct phase (solid line) and the same embedded V films shown in Fig. 5 (dashed and dotted lines). Squares, circles, and crosses give the transmission coefficient for alternative interface configurations with none, one, and two VO layers, respectively. Only the GGA results are displayed.

tion, and hence, the transport properties of the films are described by their ballistic conductance. ${ }^{18}$ The resistivity is then given by the inverse of the conductance, the latter being proportional to the transmission coefficient across a section of the film and at the Fermi energy, $T\left(E_{F}\right)$. We employ standard Green's function techniques in order to calculate $T\left(E_{F}\right),{ }^{19}$ preserving the DFT level of accuracy by using the SIESTA Hamiltonian matrix elements obtained in the previous section to construct the system's Green's functions. ${ }^{20}$ Increased $k$ samplings along the (010) direction, a small imaginary part of the energy, $\delta=1 \mathrm{meV}$, and a $10 \mathrm{meV}$ energy window around the Fermi energy were used in the conductance calculations. We also verified that modifying these values within reasonable limits did not change the conclusions.

We plot in Fig. 6 the transmission coefficient as a function of $a_{\perp}^{V}$ for the V-bct case as well as for several embedded films. Although there is a general decrease in the conductance as the interlayer $\mathrm{V}$ spacing becomes smaller, deviations from the average value are 1-2 orders of magnitude smaller than the drop in resistivity experimentally measured. The behavior is common to all the $\mathrm{V}$ films, all of them attaining similar conductance values. Hence, the interface structure and/or stoichiometry seems not responsible for the resistivity drop. The strong discrepancy with the experimental behavior implies that the ballistic assumption is not appropriate for our films and, therefore, their transport properties should be dominated by in-plane defects, i.e., the transport is in a diffusive regime.

\section{FINAL DISCUSSION}

From the measurements and the above analysis regarding the resistivity of the $\mathrm{V}$ films, we may conclude that our samples are not defect-free along the in-plane direction and that the defect concentration decreases with increasing deposition temperature. Since interface effects as well as the presence of $\mathrm{H}$ in the thin $\mathrm{V}$ films have been excluded, the only possible mechanism that could explain the resistivity drop is the reduction of the density of defects in the films with increasing growth temperature. Experimentally, however, there is no appreciable dependence of crystalline coherence length, mosaic spread, surface roughness, or any other structural parameter with the deposition temperature. However, we can 
indirectly, at least in a qualitative approach, discern the difference in the structural quality of the films by measuring the residual resistance ratio (RRR) (measured between RT and $10 \mathrm{~K}$ ) for the different structures. The RRR parameter has been recently used to determine the structural quality in ultrathin films via electrical transport characterization. ${ }^{21}$ By reducing the temperature, all sources of electrical resistivity besides defects (i.e., electronic or lattice vibrations) are frozen out, therefore observing a stronger decrease in resistivity in a high structural quality film than in an equivalent film with more structural defects. In our specific case, we experimentally observe an increase in the RRR values with $\mathrm{V}$ deposition temperature, pointing to a gradual and reasonable improvement in structural quality with deposition temperature due to a reduction in the number of defects. Regarding the nature of the defects, Ikuhara and Pirouz ${ }^{22}$ have estimated the critical thickness for the appearance of misfit dislocations in the $\mathrm{V} / \mathrm{MgO}(001)$ system to be $1.9 \mathrm{~nm}$, even though experimentally they do not observe such dislocations in $5 \mathrm{~nm}$ thick $\mathrm{V}$ films. Therefore, we cannot presume the existence of dislocations in our systems. Still, defects such as buried steps and kinks, as well as a certain amount of disorder, may be expected.

We next address the, in principle, counterintuitive observed volume compression for deposition at high temperatures. The calculations presented in the previous section have shown that the $\mathrm{V}$ films tetragonally distorted stabilize with an interlayer spacing $a_{\perp}^{V}$, which corresponds to a contracted $\mathrm{V}$ atomic volume, in agreement with the experimental results for high deposition temperatures. Therefore, the effect of temperature is to produce some degree of annealing of the film, driving the system to its most stable (volume contracted) state. One may still ask what is the origin of the expanded volume for the $\mathrm{V}$ films grown at lower temperatures. The bombardment of the growing film by neutral atoms reflected off the target ${ }^{23}$ is known to induce inhomogeneous disorder that, in turn, can give rise to a volume expansion. ${ }^{24}$ This disorder is maximum for RT depositions and, therefore, a maximum volume expansion may be expected at this temperature. Another possible mechanism for the volume expansion is the well-known residual stress of thin films produced by a sputtering process ${ }^{25-27}$ Probably the combination of both disorder and some amount of residual stress is responsible for the expansion of the films.

\section{CONCLUSIONS}

In conclusion, we have presented a study on the evolution of the resistivity of ultrathin $\mathrm{V}$ layers as a function of lattice parameter modification. Our results confirm and extend the studies presented by Meded et al.. In particular, we show that the unit volume of ultrathin $\mathrm{V}$ films can be monitored by tuning the growth temperature of the films without the need of $\mathrm{H}$ incorporation. The resulting unit volume changes (expansion and compression) have a drastic impact on the resistivity that is higher for increasing volume. The changes in resistivity are of the same order as those resulting from the incorporation of $\mathrm{H}$. The theoretical calculations demonstrate that the $\mathrm{V}$ lattice distortion and the associated resistivity drop are not related to changes at the $\mathrm{V} / \mathrm{MgO}(001)$ interface, but arise from a decrease in the density of defects within the $\mathrm{V}$ films with growth temperature. Our study highlights the difficulty of determining with high precision the degree of crystallinity of buried thin films despite the fact that the concentration of defects turns out to be crucial to the film transport properties.

\section{ACKNOWLEDGMENTS}

Work performed with partial financial support from the Spanish Commission of Science and Technology. Y.H. thanks the "Ramón y Cajal" program and Consejo Superior de Investigaciones Científicas (CSIC) for financial support. J.C. acknowledges financial support from the Spanish MCyT under Contracts Nos. NAN2004-09125-C07-06 and MAT2007-66719-C03-02. A.C. and Y.H. acknowledge financial support from the Spanish MCyT under Contract No. MAT2005-05524-C02. Elisa Román is gratefully acknowledged for stimulating scientific discussions. The Comunidad Autónoma de Madrid (CAM) under project number S-0505/ MAT/0194 NANOMAGNET is also acknowledged.
${ }^{1}$ J. Soler, J. M. Lopez Nieto, J. Herguido, M. Menéndez, and J. Santamaría, Catal. Lett. 50, 25 (1998).

${ }^{2}$ O. Rubio, J. Herguido, and M. Menéndez, Chem. Eng. Sci. 58, 4619 (2003).

${ }^{3}$ R. Vidal-Michel and K. L. Hohn, J. Catal. 221, 127 (2004).

${ }^{4}$ V. Uzdin, K. Westerholt, H. Zabel, and B. Hjörvarsson, Phys. Rev. B 68, 214407 (2003).

${ }^{5}$ H. Schiechl and A. Winkler, Fresenius' J. Anal. Chem. 371, 342 (2001).

${ }^{6}$ G. Dietrich, K. Dasgupta, S. Kuznetsov, K. Liitzenkirchen, L. Schweikhard, and J. Ziegler, Int. J. Mass Spectrom. Ion Process. 157/158, 319 (1996), and references therein.

${ }^{7}$ V. Meded, S. Olsson, P. Zahn, B. Hjövarsson, and S. Mirbt, Phys. Rev. B 69, 205409 (2004).
${ }^{8}$ P. Hohenberg and W. Kohn, Phys. Rev. 136, B864 (1964); W. Kohn and L. J. Sham, Phys. Rev. 140, A1133 (1965).

${ }^{9}$ C. Martínez Boubeta, J. L. Costa-Krämer, and A. Cebollada, J. Phys.: Condens. Matter 15, R1123 (2003).

${ }^{10}$ Y. Huttel, E. Navarro, and A. Cebollada, J. Cryst. Growth 273, 474 (2004).

${ }^{11}$ I. Tanaka, M. Mizuno, S. Nakajyo, and H. Adachi, Acta Mater. 46, 6511 (1998).

${ }^{12}$ T. Burkert, A. Miniotas, and B. Hjövarsson, Phys. Rev. B 63, 125424 (2001).

${ }^{13}$ E. Román, Y. Huttel, M. F. López, R. Gago, A. Climent-Font, A. Muñoz-Martín, and A. Cebollada, Surf. Sci. 600, 497 (2006).

${ }^{14}$ N. Troullier and J. L. Martins, Phys. Rev. B 43, 1993 (1991).

${ }^{15}$ J. M. Soler, E. Artacho, J. Gale, A. García, J. Junquera, P. Orde- 
jón, and D. Sánchez-Portal, J. Phys.: Condens. Matter 14, 2745 (2002).

${ }^{16}$ D. M. Ceperley and B. J. Alder, Phys. Rev. Lett. 45, 566 (1980).

${ }^{17}$ J. P. Perdew, K. Burke, and M. Ernzerhof, Phys. Rev. Lett. 77, 3865 (1996).

${ }^{18}$ S. Datta, Electronic Transport In Mesoscopic Systems (Cambridge University Press, New York, 1995).

${ }^{19}$ J. Cerdá, M. A. Van Hove, P. Sautet, and M. Salmeron, Phys. Rev. B 56, 15885 (1997); see also www.icmm.csic.es/jcerda

${ }^{20}$ C. Rogero, C. Koitzsch, M. E. González, P. Aebi, J. Cerdá, and J. A. Martín-Gago, Phys. Rev. B 69, 045312 (2004).

${ }^{21}$ M. Gutsche, H. Kraus, J. Jochum, B. Kemmather, and G.
Gutekunst, Thin Solid Films 248, 18 (1994).

${ }^{22}$ Y. Ikuhara and P. Pirouz, Microsc. Res. Tech. 40, 206 (1998).

${ }^{23}$ H. Tokura, B. Window, D. Neely, and M. Swain, Thin Solid Films 253, 344 (1994).

${ }^{24}$ S. R. Phillpot, D. Wolf, and S. Yip, MRS Bull. 15, 38 (1990).

${ }^{25}$ C. Hudson and R. E. Somekh, J. Vac. Sci. Technol. A 14, 2169 (1996).

${ }^{26}$ A. Misra and M. Nastasi, J. Mater. Res. 14, 4466 (1999).

${ }^{27}$ Y. G. Shen, Y. W. Mai, Q. C. Zhang, D. R. McKenzie, W. D. McFall, Q. C. Zhang, D. R. McKenzie, and W. D. McFall, J. Appl. Phys. 87, 177 (2000). 